\title{
Urban background noise mapping: the general model
}

\author{
W. Wei ${ }^{1}$, D. Botteldooren ${ }^{1}$, T. Van Renterghem ${ }^{1}$, M. Hornikx ${ }^{2,5}$, J. Forssén ${ }^{2}$, E. Salomons ${ }^{3}$, \\ and M. Ögren ${ }^{4}$ \\ ${ }^{1}$ Department of Information Technology, Ghent University, Sint-pietersnieuwstraat 41, 9000 \\ Ghent, Belgium, weigang@intec.ugent.be \\ ${ }^{2}$ Department of Civil and Environmental Engineering, Chalmers University of Technology, \\ Gothenburg, Sweden, \\ ${ }^{3}$ TNO Urban Environment and Health, Delft, The Netherlands, \\ ${ }^{4}$ Department of Environmental and Traffic Analysis, VTI, Gothenburg, Sweden, \\ ${ }^{5}$ Building Physics and Services, Eindhoven University of Technology, Eindhoven, the \\ Netherlands.
}

\begin{abstract}
Surveys show that inhabitants of dwellings exposed to high noise levels benefit from having access to a quiet side. However, current practice in noise prediction often underestimates the noise levels at a shielded façade. Multiple reflections between façades in street canyons and inner yards are commonly neglected and façades are approximated as perfectly flat surfaces yielding only specular reflection. In addition, sources at distances much larger than normally taken into account in noise maps might still contribute significantly. Since one of the main reasons for this is computational burden, an efficient engineering model for the diffraction of the sound over the roof tops is proposed, which considers multiple reflections, variation in building height, canyon width, façade roughness and different roof shapes. The model is fitted on an extensive set of full-wave numerical calculations of canyon-to-canyon sound propagation with configurations matching the distribution of streets and building geometries in a typical historically grown European city. This model allows calculating the background noise in the shielded areas of a city, which could then efficiently be used to improve existing noise mapping calculations. The model was validated by comparison to long-term measurements at 9 building façades whereof 3 were at inner yards in the city of Ghent, Belgium. At shielded façades, a strong improvement in prediction accuracy is obtained.
\end{abstract}

\section{Introduction}

Several researchers found that inhabitants of dwellings exposed to road traffic noise levels can benefit from having access to a quiet side 1, 2, 3, 4. The European Environmental Noise Directive (END) specifies that a quiet side is present if the noise level at the shielded façade is at least $20 \mathrm{~dB}$ lower than the noise level at the most exposed façade of the dwelling (Directive 2002/49/EC). However, there is still some debate about accurately defining a quiet side (e.g. [5]). In typical European cities, many enclosed shielded courtyards and parks exist that can provide such quiet areas. Notwithstanding the 
lack of a good definition, research on quiet sides and its implementation in urban planning also suffers from a lack of accuracy in commonly used noise mapping when it comes to predicting noise levels in urban shielded areas. The EU is currently renewing its guidelines for methods to be used in noise mapping, yet the lack of accuracy of noise mapping in shielded areas is mainly due to the choices made during implementation and application of the methods. Typically, the underestimation of the noise level at such shielded places, is caused by limiting the number of reflections in streets and yards and by neglecting contributions of distant sources that could become dominant. To solve these problems, simplified theoretical models, such as the "flat city model" and the "equivalent source model(ESM)", were developed to predict the noise level in shielded courtyards [6, 7. However, these models need further improvement. For example, the coupling between the sound field inside a street canyon and the propagation above the roofs can depend on the difference in height of the buildings forming the street canyon. Moreover, the ESM is computationally too costly to cover a whole city. In this paper, an efficient engineering model for background noise mapping is proposed that is inspired by the concept of the "flat city model" and a new approximation to more advanced diffraction formulas. The coefficients of the proposed engineering model are fitted on an extensive set of $2 \mathrm{D}$ simulations of canyon-to-canyon sound propagation, which are based on finite-difference-time-domain (FDTD) method [8, 9]. The effect of multiple reflections, variation in building height, canyon width, building façade roughness, finite impedance and roof shape is taken into account. The proposed engineering model is designed to complement the noise map calculated by $2.5 \mathrm{D}$ methodologies in which the diffraction over buildings due to reflections between canyons may not be sufficiently considered. The direct field, reflection in the horizontal plane as well as diffraction around vertical edges is assumed to be accounted for by the "parent" model (e.g. following the CNOSSOS-EU methodology. The proposed extension calculates the contribution to the noise level caused by all sources that are shielded by at least one building in the vertical plane. In this work, a building is a construction of at least $4 \mathrm{~m}$ high and at least $5 \mathrm{~m}$ wide; conventional noise barriers are expected to be correctly included in the "parent" model. The resulting "background" noise level should be added to the level obtained using the "parent" model. With this approach the national and international standard methods currently in use can still be applied. For every contributing source the suggested procedure for calculating the "background" noise level at the shielded location reads:

$$
\begin{gathered}
L_{p b}=10 \log _{10}\left(10^{0.1 L_{p d b}}+10^{0.1 L_{p, s c a t t e r}}\right) \\
L_{p d b}=L_{W}-A_{\text {free }}-A_{d i f f r}-A_{\text {inter }} \\
A_{\text {diffr }}=-10 \log _{10}\left(10^{-0.1 A_{b a r}}+10^{-0.1 A_{\text {can }}}\right)
\end{gathered}
$$

where,

- $L_{p b}=$ the "background" sound level excluding the diffraction around the vertical edges and excluding the diffraction over conventional noise barriers $[\mathrm{dB}]$.

- $L_{p d b}=$ the contribution to the "background" level in still, homogeneous atmosphere [dB].

- $L_{W}=$ sound power level per octave band of a point source representing part of the traffic, no directivity is taken into account since multiple sources will contribute to the shielded level as 
well as multiple reflections from various directions $[\mathrm{dB}]$.

- $A_{d i f f r}=$ the attenuation caused by the building, which is limited by diffraction and multiple reflections over the building $[\mathrm{dB}]$.

- $A_{\text {free }}=3 \mathrm{D}$ free field divergence $[\mathrm{dB}]$.

- $A_{b a r}=$ the attenuation by the building(s) cutting the direct path between source and receiver limited by diffraction over the building roof, including the effect of the ground. Only the direct diffraction path without reflections in the canyon is considered in this term [dB].

- $A_{c a n}=$ the attenuation of the sound following a path between source and receiver including at least one reflection in the source and/or receiver canyon. If canyons are present, this term quickly dominates $A_{b a r}$ and thus determines $A_{d i f f r}[\mathrm{~dB}]$.

- $A_{\text {inter }}=$ additional attenuation caused by diffraction at intermediate canyons $[\mathrm{dB}]$.

- $L_{p, \text { scatter }}=$ the contribution to the background sound level caused by scattering from atmospheric turbulence $[\mathrm{dB}]$ [10.

Atmospheric absorption is not included as a separate term in this model as it is implicitly included in the $A_{\text {can }}$ term, where the absorption was added by post-processing the FDTD simulation results. One of the major assumptions for the model is that the sound propagation in $3 \mathrm{D}$ can be calculated by the summation of many $2 \mathrm{D}$ sections. This so called 2.5D approach is quite common and forms e.g. the basis of the Harmonoise reference model [11] and the CNOSSOS-EU methodology [12. Also, the full wave numerical model used to extract the coefficients in the proposed equations cannot be used for 3D simulations due to computational cost. Therefore, also for the reference calculation, line sources are split into many emission points and all contributions are summed. In this approach, façades are "twisted" so their faces become normal to the line connecting source-receiver [13]. It can be shown from numerical simulations that the error caused by using the twisted angle approach is reasonably small [14]. A correction for 3D free field spreading of the contributions of reflections is taken into account. A second important assumption is that wind and temperature gradients are not included in the $A_{b a r}$ and $A_{c a n}$ terms. For the $A_{\text {inter }}$ term meteorological effects (except scattering) are considered implicitly(see further) since downwind refraction over larger distances may have a noticeable effect.

This paper is organized as follows. In section 2, the configurations and setups of the simulations that are used for fitting coefficients in the engineering model are introduced. In section 3, the attenuation terms (A-terms) are studied in detail. In section 4, the calculated background noise levels are compared to long-term measurements at 9 locations in the city of Ghent, Belgium. The latter comparison includes the contribution from turbulence scattering. The engineering model developed for calculating this contribution can be found in a companion paper [10].

\section{Simulation configurations and setups}

The simulations cover different widths of source canyons, receiver canyons and intermediate buildings, as well as different building heights. Distributions of these parameters for a typical historically grown European city, are extracted from a GIS-building layer for the city of Ghent. The distribution of the projected canyon and building widths along each source-receiver line is shown in figure 1. Note that the width is defined along a line that is not necessarily orthogonal to the building façade, which is 


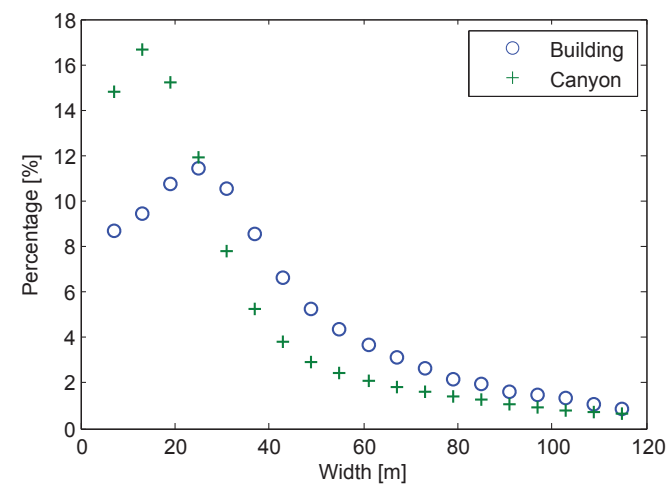

(a) Distribution

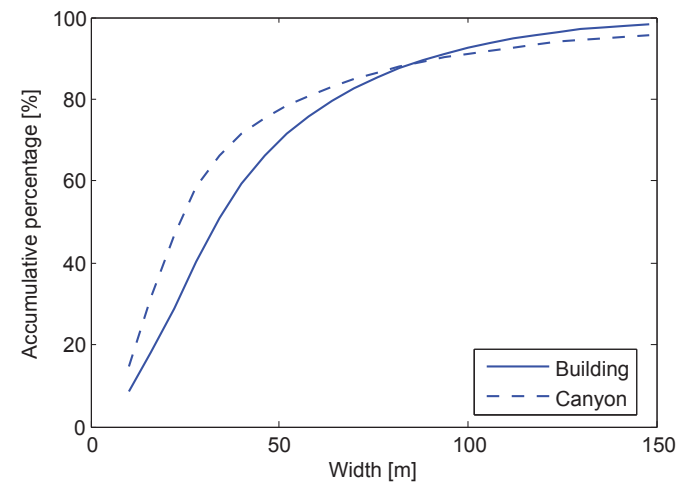

(b) Cumulative probability

Figure 1: Distribution(a) and cumulative probability(b) of the projected building and canyon width in the city of Ghent, Belgium. In the simulations, the widths of buildings and canyons are limited to 42 $\mathrm{m}$.

compatible with the point-to-point model that is proposed. $72 \%$ of the projected buildings widths and $78 \%$ of the canyons are less than $50 \mathrm{~m}$ wide. Besides, the most frequent projected widths of the buildings and canyons are $24 \mathrm{~m}$ and $12 \mathrm{~m}$, respectively. The full wave numerical simulations on which the engineering model is based have been limited to canyon and building widths between $4.8 \mathrm{~m}$ and $42 \mathrm{~m}$. The heights of the buildings are varied from 4 to $16 \mathrm{~m}$. The building façades are modeled in a realistic way by assigning different materials and making the façade surface irregular to allow for the build-up of a diffuse sound field in the city canyons, which was shown to be an essential aspect of urban sound propagation [15. The road surface is modelled as perfectly reflecting both in source and receiver canyon. In these simulations, the normalized impedance of windows and brick walls are taken as $Z_{n}=77$ and $Z_{n}=10$ respectively. Receivers are located along the façade and across the canyons. A typical simulation configuration is shown in figure 2, where, $W_{s}, W_{i}, W_{r}$ are the width of the source canyon, intermediate building and receiver canyon respectively. $H_{i}$ is the height of the building in the direct sound path, and $H_{s}, H_{r}$ are the heights of the buildings flanking the source and receiver canyon respectively. Since the sound waves travel a longer distance because of multiple reflections, the air will absorb more sound energy than during direct propagation between source and receiver. The effect of the air absorption, with $T=10^{\circ} \mathrm{C}$ and Humidity $=70 \%$, is added to the simulated impulse response using the approach proposed in references [16, 17.

The multiple reflection effect will change with the relative location of the source and receiver, the height of the buildings and the width of the canyon and buildings. In total, 565 configurations with combinations of these parameters were simulated.

The excess attenuation caused by screening and ground effects was proven not to be affected too much by source type in the far field [18. However, in the case of multiple reflections in a street canyon, small differences might still occur. Therefore, the time-domain response is multiplied by $1 / \sqrt{c t}$ to approximately translate the line source propagation to point source propagation [19]. In our data post-processing, this technique is used to approximate point source propagation from canyon to canyon. 


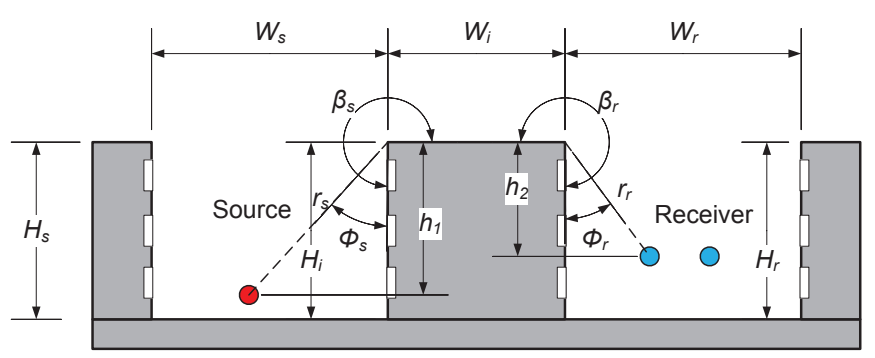

Figure 2: A typical simulation configurations, where $W_{s}, W_{i}$ and $W_{r}$ are the width of source canyon, intermediate building and receiver canyon; $H_{s}, H_{i}$ and $H_{r}$ are the height of the left, intermediate and right building; $h_{1}$ and $h_{2}$ are the distance from the source or receiver to the top of the building; $\phi_{s}$ and $\phi_{r}$ are the angle between the building façade and the connecting line from the source or receiver to the diffraction edge; $\beta_{s}$ and $\beta_{r}$ are the outside angle of the building which equals $3 \pi / 2$ in this study.

\section{Analysis of attenuation terms: $A_{b a r}, A_{c a n}$, and $A_{\text {inter }}$}

The attenuation is resolved as separate parts $A_{d i f f r}$ and $A_{\text {inter }}$, where $A_{d i f f r}$ is calculated by the sum $A_{b a r}$ and $A_{c a n}$, as $-10 \log _{10}\left(10^{-0.1 A_{b a r}}+10^{-0.1 A_{c a n}}\right)$. $A_{b a r}$ and $A_{c a n}$ will be formulated in section 3.1 and section 3.2 respectively. $A_{\text {inter }}$ will be introduced in section 3.3 .

\section{1 $A_{b a r}$}

$A_{b a r}$ is the attenuation of a thick barrier including the presence of the ground. In absence of canyons and in case of a flat roof, it is the only remaining term. In this study $A_{b a r}=A_{b a r, f l a t},+A_{b a r, \text { roof }}$, where, $A_{b a r, f l a t}$ is the attenuation of a rigid barrier with flat roof; $A_{b a r, \text { roof }}$ is the correction of the roof shape in $\mathrm{dB}$.

\subsection{1 $A_{b a r, f l a t}:$ rigid barrier with flat roof}

In most noise mapping standards (including CNOSSOS-EU), the ISO9613-2 diffraction formula or similar is used to calculate $A_{b a r, \text { flat }}$. By comparing with an in-situ long-term measurements [20] and FDTD simulations, it was found that using the ISO standard to calculate $A_{b a r}$ underestimates the attenuation considerably. Therefore, a more accurate but still computational efficient approximation is needed. In a first step, ground reflection is ignored. According to the literature [21, 22, 23, 24, $A_{\text {bar,flat }, 0}$ can be expressed with high accuracy by equation (4):

$$
A_{\text {bar }, \text { flat }, 0}=-10 \log _{10}\left\{\left(\frac{R}{L}\right)^{2}\left[f^{2}\left(X_{1}\right)+g^{2}\left(X_{1}\right)\right]\left[f^{2}\left(X_{2}\right)+g^{2}\left(X_{2}\right)\right]\right\}
$$

where, $X_{1}=Y_{s}$ and $X_{2}=B Y_{r}$ when $Y_{s}>Y_{r} ; X_{1}=B Y_{s}$ and $X_{2}=Y_{r}$ when $Y_{s}<Y_{r}$. $Y_{s}$ and $Y_{r}$ are functions of geometrical positions and diffraction angles. $Y_{s}=\gamma_{s} M_{\nu s}\left(\beta_{s}-\phi_{s}\right)$, $Y_{r}=\gamma_{r} M_{\nu r}\left(\beta_{r}-\phi_{r}\right), \gamma_{s}=\sqrt{2 r_{s}\left(W_{i}+r_{r}\right) /(\lambda L)}, L=\sqrt{\left(r_{s}+r_{r}+W_{i}\right)^{2}+\left(z_{s}-z_{r}\right)^{2}},\left(z_{s}=z_{r}\right.$ in the two dimensional case considered here), $R$ is the distance between source and receiver, $B=$ $\sqrt{W_{i}\left(W_{i}+r_{s}+r_{r}\right) /\left[\left(W_{i}+r_{s}\right)\left(W_{i}+r_{r}\right)\right]}$ and $M_{\nu s}(\theta)=\frac{\cos (\nu \pi)-\cos (\nu \theta)}{\nu \sin (\nu \pi)}, \nu_{s}=\pi / \beta_{s}$ and $\nu_{r}=\pi / \beta_{r}$. Definitions of parameters are shown in figure 2, $f(Y)$ and $g(Y)$ are functions of Fresnel integrals $C$ 
and $S$ [21]:

$$
\begin{aligned}
& f(Y)=\left[\frac{1}{2}-S(Y)\right] \cos \left(\frac{1}{2} \pi Y^{2}\right)-\left[\frac{1}{2}-C(Y)\right] \sin \left(\frac{1}{2} \pi Y^{2}\right) \\
& g(Y)=\left[\frac{1}{2}-C(Y)\right] \cos \left(\frac{1}{2} \pi Y^{2}\right)+\left[\frac{1}{2}-S(Y)\right] \sin \left(\frac{1}{2} \pi Y^{2}\right)
\end{aligned}
$$

The combination $f^{2}+g^{2}$ needed in equation (4) simplifies since the cosine and sine functions cancel out, reducing the expression to:

$$
f^{2}(Y)+g^{2}(Y)=[0.5-C(Y)]^{2}+[0.5-S(Y)]^{2}
$$

where $Y$ is the input argument. For a noise mapping model, calculating the Fresnel integrals is computationally too costly, so an approximation is needed.

For this, it is first observed that the distances involved in the formula for diffraction over building, and in particular $W_{i}$, are generally large compared to the wavelength. Thus, $\gamma$ will be large. If source and receiver heights are much lower than the building height, $\beta-\phi$ will remain larger than $\pi$ and it can be verified that $M_{\nu}$ is not smaller than one. For these cases, the input argument $Y$ satisfies $Y \gg 0$. For large arguments, the Fresnel integrals can be approximated by [25]:

$$
\begin{aligned}
& C(Y) \approx 0.5+\frac{1}{\pi Y} \sin \left(\frac{\pi}{2} Y^{2}\right) \\
& S(Y) \approx 0.5-\frac{1}{\pi Y} \cos \left(\frac{\pi}{2} Y^{2}\right)
\end{aligned}
$$

Inserting equation (8) and (9) into equation $(7)$ results in a very simple form for $f^{2}+g^{2}$ :

$$
f^{2}(Y)+g^{2}(Y)=\frac{1}{(\pi Y)^{2}}
$$

However, when the source or observer are in the extension of the plane of the roof, the angle difference $\beta-\phi$ approaches $\pi$ and $M_{\nu}$ approaches zero which makes $S(Y), C(Y)$ and $f^{2}(Y)+g^{2}(Y)$ become singular. To avoid this strong singularity while keeping the error at larger $\mathrm{X}$ limited, a small constant is added to the numerator and denominator. The value of this constant is obtained by requiring that the error it introduces is small for typical values of $X_{1}$ and $X_{2}$ found in a typical city (see Figure (4p). In addition the approximation minimizes the error over the whole range of angles (Figure (3)). The following approximation of equation (10) is proposed:

$$
f^{2}(Y)+g^{2}(Y)=\left(\frac{0.37}{Y+0.37}\right)^{2}
$$

Thus, equation (4) is simplified to:

$$
A_{\text {bar }, \text { flat }, 0} \approx-10 \log _{10}\left[\left(\frac{R}{L}\right)^{2}\left(\frac{0.37}{X_{1}+0.37}\right)^{2}\left(\frac{0.37}{X_{2}+0.37}\right)^{2}\right]
$$

When $Y_{s}>Y_{r}, X_{1}=\sqrt{\frac{6 r_{s}\left(W_{i}+r_{r}\right)}{\lambda\left(r_{s}+W_{i}+r_{r}\right)}}\left|-0.5+\cos \left(\frac{2}{3} \phi_{s}\right)\right|, X_{2}=\sqrt{\frac{6 r_{r} W_{i}}{\lambda\left(W_{i}+r_{r}\right)}}\left|-0.5+\cos \left(\frac{2}{3} \phi_{r}\right)\right|$; when $Y_{s}<Y_{r}, X_{1}=\sqrt{\frac{6 r_{s} W_{i}}{\lambda\left(r_{s}+W_{i}\right)}}\left|-0.5+\cos \left(\frac{2}{3} \phi_{s}\right)\right|, X_{2}=\sqrt{\frac{6 r_{r}\left(W_{i}+r_{s}\right)}{\lambda\left(r_{s}+W_{i}+r_{r}\right)}}\left|-0.5+\cos \left(\frac{2}{3} \phi_{r}\right)\right|$. 


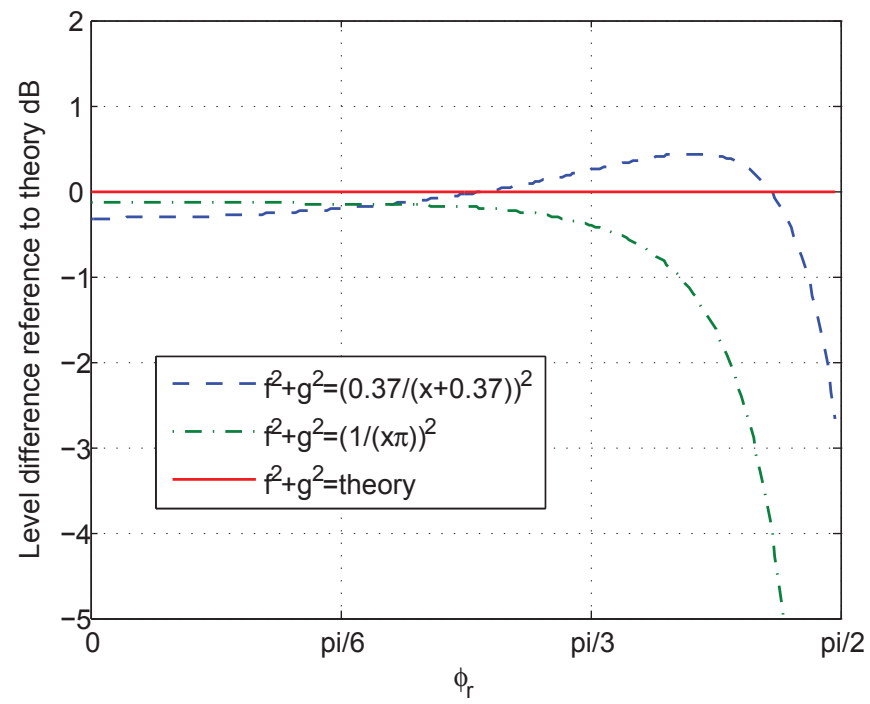

Figure 3: Error estimation of $f^{2}+g^{2}$ compared with the theoretical values. In this case, $W_{i}=10 \lambda, r_{s}=$ $r_{r}=10 \lambda, \beta_{s}=\beta_{r}=\frac{3 \pi}{2}, \phi_{s}=\frac{\pi}{4}, \phi_{r}$ increases from 0 to $\pi / 2$

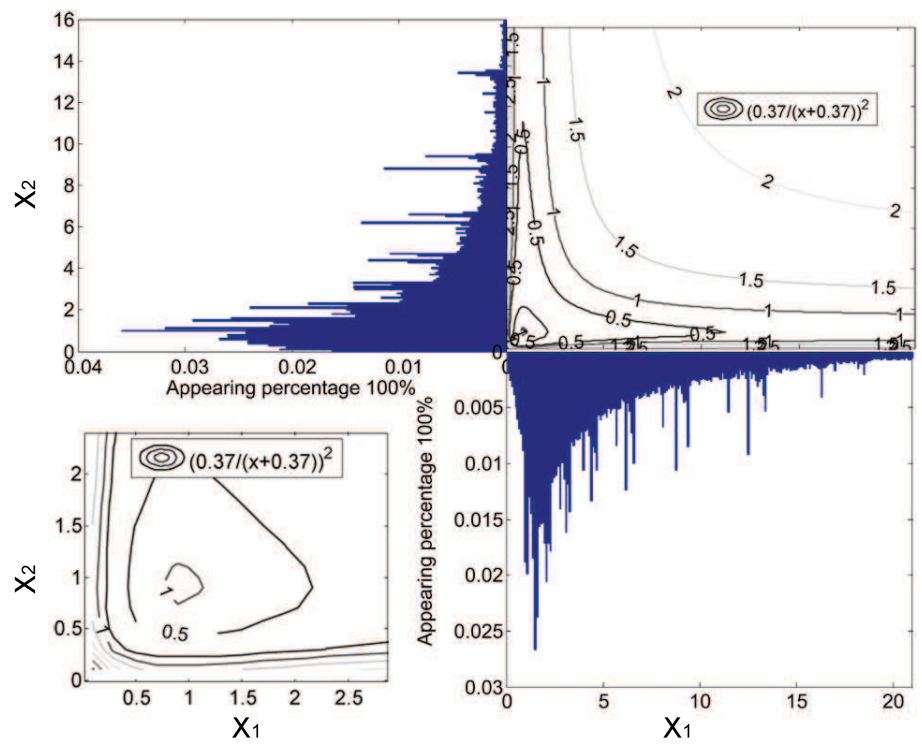

Figure 4: Error estimation reference to the theoretical solution, where " $\left(\frac{0.37}{X+0.37}\right)^{2}$ " indicates the equation (11). Every line indicates $0.5 \mathrm{~dB}$ difference. 
Figure 3 illustrates how, for a typical urban sound propagation case, the large argument approximation and the proposed approximation for the Fresnel integrals differ from the accurate calculation. Although there is a small increase in inaccuracy for the proposed approximation when $\phi_{r}$ is very small, a strong benefit can be observed for $\phi_{r}>\pi / 3$. Even when $\phi_{r}=\pi / 2$, there is still less than $3 \mathrm{~dB}$ deviation. Specifically, at $X=0$ the approximate formula gives 1 , however, knowing that $\mathrm{C}$ and $\mathrm{S}$ become zero at $X=0$, the actual value should be 0.5 , which implies a $3 \mathrm{~dB}$ error. It should however be kept in mind that this situation will only occur for very few of the source receiver paths contributing to the overall noise level. To further illustrate the fitness of the proposed approximation for diffraction over buildings, the distribution of the input $X$ is extracted for the city of Ghent and plotted together with error contours in figure 4. At the $X_{1}$ and $X_{2}$ combinations where the distribution peaks, the error introduced by using equation $(12)$ is particularly small and it stays below $1.5 \mathrm{~dB}$ for all combinations that have a significant probability of occurrence.

With this simplification, the Fresnel integrals in equation 7 are canceled out and only the geometrical parameters remains, which reduces computing time considerably and makes it easier to implement. It is suggested to include contributions from the image sources due to ground reflections explicitly as a rule. Since the source height for road traffic noise applications is usually very low, the diffraction term does not differ significantly between the path from the original source and from the image source and the calculation can be simplified by assuming that $A_{b a r, \text { flat }}$ is the same as $A_{b a r, f l a t, 0}$. The total $A_{b a r, f l a t}$ can be obtained by summing up the contribution of paths "source $\rightarrow$ receiver" $A_{b a r, f l a t, 0}$, "image source $\rightarrow$ receiver" $A_{b a r, f l a t, 1}$, "source $\rightarrow$ image receiver" $A_{b a r, f l a t, 2}$ and "image source $\rightarrow$ image receiver"

\subsection{2 $A_{b a r, r o o f}:$ correction of roof shape}

In some European city centers, gabled roofs are very common. The sound waves propagating over an idealized gabled roof may be diffracted once, twice or three times before reaching the observer. It should be noted that roofs may be more complicated and diffraction may result in a wide range of significantly different sound attenuation [26]. The effect of roof shape depends on the source and receiver position, the angle of the roof and the building height. According to the statistics for Ghent (as a typical old European city), the most common width of canyons and buildings is $12 \mathrm{~m}$ and $24 \mathrm{~m}$ respectively, and the mean height of the buildings is $10.9 \mathrm{~m}$. If the height of the roof is assumed to be $4.5 \mathrm{~m}$, then most of the sources and receivers below $4.5 \mathrm{~m}$ high would be located inside the shadow region where the sound wave has to diffract three times to reach the receiver at the other side of the building, as shown in figure 5. Under this condition, $A_{b a r, \text { roof }}$ is linearly correlated to $A_{b a r, \text { flat }}$. To simplify the calculation of the roof, $A_{b a r, \text { roof }}$ is rewritten as a linear function of $A_{b a r, f l a t}$ :

$$
A_{\text {bar, roof }}=q_{0} A_{\text {bar, flat }}-q_{1}
$$

Fitting on 1788 numerical calculations by least squares method yields values of, $q_{0}=0.27$ and $q_{1}=3.4$, with a mean squared error of the fit equal to $3.0 \mathrm{~dB}$ for equation 13 . The fitting database covers building heights from $6 \mathrm{~m}$ to $16 \mathrm{~m}$, building widths from $10 \mathrm{~m}$ to $160 \mathrm{~m}$ and all roof heights are $4.5 \mathrm{~m}$. Figure (6) shows the accuracy of the fit for a particular situation.

If a source canyon or a receiver canyon is present, the image source or the image receiver would most probably lie outside the three-diffraction region, which means that the effect of roof shape on 


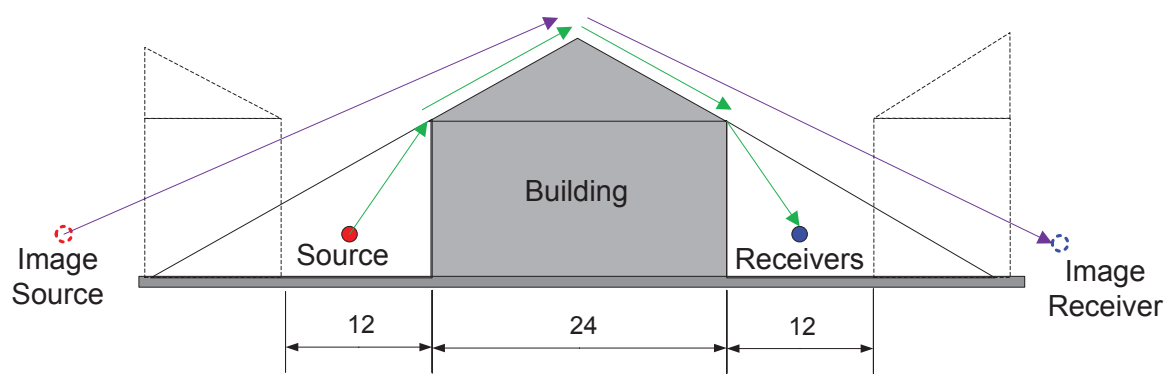

Figure 5: Effect of roof shape.

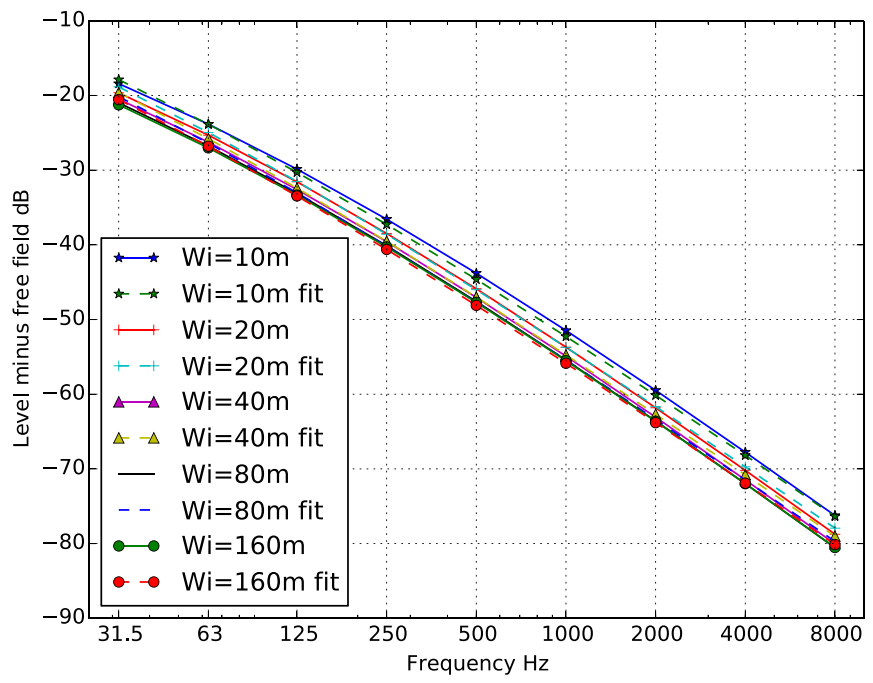

Figure 6: Accuracy of the fitted and analytical curve. The curve labeled " $W_{i}=10 \mathrm{~m}$ " indicates the exact values, while curve labeled " $W_{i}=10 \mathrm{~m}$ fit" indicates the approximated values obtained using equation (13). The height of the building is $12 \mathrm{~m}$. The source is $5 \mathrm{~m}$ to the façade and the distances from receivers to façade are $0.1,0.5,1,2,3,5 \mathrm{~m}$ respectively. 
the multiple-reflection path would probably be much more important. The roof effect in such cases is discussed in detail in section(3.2) as $A_{\text {can,roof }}$.

\section{$3.2 A_{c a n}$}

$A_{c a n}$ is the attenuation of the sound following a path between source and receiver including at least one reflection in the source and/or receiver canyon. $A_{c a n}=A_{c a n, \text { flat }}+A_{\text {can,roof }}$, where $A_{\text {can,flat }}$ is the extra attenuation in case of a flat roof on the intermediate building; $A_{\text {can,roof }}$ is a correction accounting for a gabled roof shape.

An analytic formulation for the additional effect of the canyons has to fulfill some requirements: 1) if the height of the outer buildings goes to zero, the term should vanish; 2) if the outer buildings become much higher than the screening building, $A_{\text {can }}$ should saturate when further increasing the outer building height.

\subsubsection{Contribution of multiple reflections}

Multiple reflections, occurring at the façades of the outer buildings and intermediate building, influence the canyon-to-canyon propagation in a different way. When $H_{s}$ or $H_{r}$ increases, the effect of multiple reflections increases monotonically at all frequencies. When $H_{i}$ increases, the effect of multiple reflections increases at one hand. At the other hand, the shielding of the middle building also increases. Similar to $A_{b a r}, A_{c a n, f l a t}$ is also frequency dependent.

\subsubsection{Formulation of $A_{\text {can, flat }}$}

Suppose the buildings are not very low and the sources are in the middle of the canyon, then an analytical form of $A_{\text {can,flat }}$ can be obtained based on image source theory. Details are described in Appendix A. By adding fitting coefficients $F(0), F(1), F(2)$ and $F(3)$ to different contributing parts, small approximation errors as well as effects of non flat façades can be reduced. The proposed analytical form thus reads::

$$
\begin{aligned}
A_{\text {can }, \text { flat }} & \approx-F(0) 10 \log _{10}\left[F(1) \frac{C_{1 s} \rho_{s}^{2} R^{2}}{\left(C_{3 s}+W_{s}\right)^{2}} 10^{0.1 L_{h s}}+F(2) \frac{C_{1 r} \rho_{r}^{2} R^{2}}{\left(C_{3 r}+W_{r}\right)^{2}} 10^{0.1 L_{h r}}\right. \\
& \left.+F(3) \frac{\rho_{s}^{2} \rho_{r}^{2} R^{2}}{\left(3.31 h_{1} / \sqrt{\lambda}+C\right)\left(3.31 h_{2} / \sqrt{\lambda}+C\right)} 10^{0.1 L_{h s}} 10^{0.1 L_{h r}}\right]
\end{aligned}
$$

where $C=1.5 W_{s}+W_{i}+1.5 W_{r}$, other parameters can be found in the appendix.

The three terms in equation (14) describe the contribution from all image sources to all image receivers. The $F(1)$ term can be interpreted as a reverberant source canyon field diffracted into the receiver canyon. As such $\left(C_{3 s}+W_{s}\right)^{2}=1 /\left(3.31 h_{1} \sqrt{W_{i} / \lambda}+1.5 W_{s}+W_{i}+r_{r}\right)^{2}$ expresses mainly the amplification due to the source canyon reverberation. A similar interpretation can be given to the $\mathrm{F}(2)$ and $\mathrm{F}(3)$ terms. If $W_{i}$ becomes very large, the whole $F(1), F(2)$ and $F(3)$ term will approach $F(1) C_{1 s} \rho_{s}^{2} 10^{0.1 L_{h s}}, F(2) C_{1 r} \rho_{r}^{2} 10^{0.1 L_{h r}}$ and $F(2) \rho_{s}^{2} \rho_{r}^{2} 10^{0.1 L_{h s}} 10^{0.1 L_{h r}}$, which implies that the effect of the source and receiver canyon become independent and are then only related to the receiver or source canyon dimension. This is also verified by numerical simulation as shown in [14. If $W_{s}$ becomes very big, the source canyon effect will vanish and similar situations can be found for the receiver canyon 


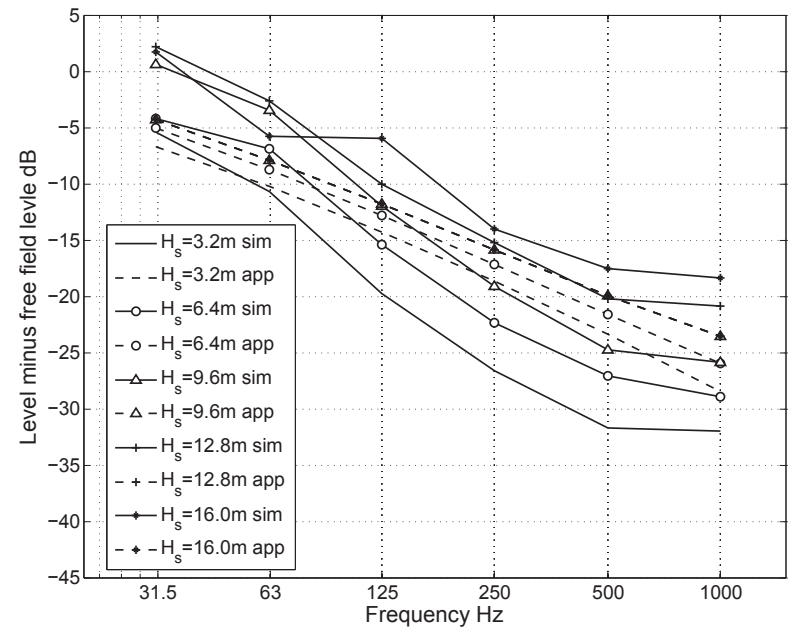

(a) Case study 1 with $H_{i}=H_{r}=W_{s}=W_{r}=9.6 \mathrm{~m}$,(b) Case study 2 with $H_{s}=H_{r}=W_{s}=W_{r}=9.6 \mathrm{~m}$, $W_{i}=10 \mathrm{~m}$, and $H_{s}$ changes

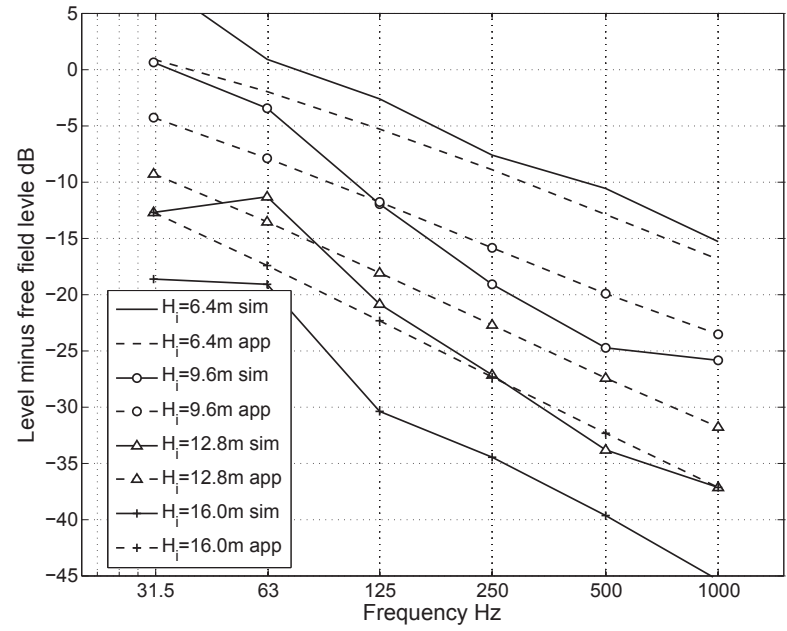

$W_{i}=10 \mathrm{~m}$, and $H_{i}$ changes

Figure 7: Comparison between FDTD simulations and the analytical approximation. In these comparisons, the source is in the center of the canyon with height of $0.5 \mathrm{~m}$. The receiver is $6.4 \mathrm{~m}$ away from the intermediate building which has a height of $4 \mathrm{~m}$.

if $W_{r}$ becomes large. If $h_{2} \rightarrow 0, L_{h r}$ becomes meaningless because of $\left(H_{r}-h_{r}\right) /\left(H_{i}-h_{r}\right)$ tending to $\infty$. This condition implies that the receiver is at the same height as the top of the shielding building and the canyon effect can then be neglected. As a result, the $F(2)$ term becomes zero. Since the source position is almost close to the ground in most cases, $h_{1}$ is expected not to tend to zero.

Based on fitting equation (14) to the database containing (20740 numerical results) with $\rho_{s}=$ $\rho_{r}=0.97$, minimizing the quadratic error, the following coefficients were found: $F(0)=1.04$, $F(1)=12.53, F(2)=21.75$ and $F(3)=0.05$. The mean squared error between $A_{\text {can,flat }}$ and the simulated results is $7.1 \mathrm{~dB}$. If both $H_{s}$ and $H_{r}$ are very large, $A_{\text {can, flat }}$ tends to a constant.

A comparison between the fitted equation and several common cases calculated by the FDTD method are shown in figure 7. Several typical configurations are depicted, showing good agreement between the fitted equation and the detailed simulations. The analytical approximation follows the increasing or decreasing trends very well, but it cannot capture the increase if $H_{s}>H_{i}$. Additionally, if $H_{s}$ is much lower than $H_{i}$, an overestimation of the reflections could be observed. The reason could be because that the diameter of ellipsoid between image source and receiver is much bigger than $H_{s}$. In this condition, the sound waves "leak" from the canyon meanwhile the model still include this part.

\subsection{3 $A_{\text {can, roof }}$}

When canyons are present, the sound reflects in these canyons and the $A_{\text {can, roof }}$ can be considered as the extra attenuation of the sum of different $A_{b a r, f l a t}$ terms with different powers and positions of image sources and receivers. However, the image sources could reach the receivers or image receivers by only one diffraction from the roof top which will significantly increase the sound power at the 
receiver positions, as show in figure 5. Additionally, this effect depends strongly on the geometrical configuration of the buildings and canyons which also differs significantly from one to another. In this study, $A_{\text {can,roof }}$ is quantified from the literature [26], where an extensive set of roof shapes was numerically studied. The general contribution of a gabled roof was around $-5 \mathrm{~dB}$. In this study, $A_{\text {can,roof }}=-5 \mathrm{~dB}$ if both a source and a receiver canyons are present and $A_{\text {can,roof }}$ increases to $-2.5 \mathrm{~dB}$ if only one canyon is present. $A_{\text {can, roof }}$ is zero on condition that the roof shape is flat.

\section{$3.3 \quad A_{\text {inter }}$}

The presence of intermediate canyons could lead to additional attenuation of sound. Since $A_{\text {can }}$ is fitted based on thick barrier simulations only, an additional correction term, $A_{\text {inter }}$, is necessary. According to the FDTD simulations, $A_{\text {inter }}$ increases approximately by $1.8 \mathrm{~dB} /$ canyon for buildings of equal height as shown in(figure 8). A previous study also shows $1.8 \mathrm{~dB} /$ canyon [35] and similar findings were reported based on the measurement data from Södermalm in Stockholm [27. As mentioned in the introduction, moderately downward refraction is assumed. At longer propagation distances, the effect of an atmospheric gradient resulting in downward refraction can be approximated by bending the ground surface upward which is comparable to lowering intermediate buildings [33. Figure (9) shows the effect of different heights of intermediate buildings. In this figure, "H" indicates the height of the high intermediate building-block and equals $9.6 \mathrm{~m}$; "L" is equal to $6.4 \mathrm{~m}$; the combination of " $\mathrm{H}$ " and " $\mathrm{L}$ " indicates the succession of the building-blocks. For example "HLLHLH" means that the sound waves propagate over $\rightarrow$ a higher building $\rightarrow$ two lower buildings $\rightarrow$ a higher building $\rightarrow$ a lower building $\rightarrow$ a higher building, and finally to the receiver. According to the simulations, the effect caused by the low buildings in between can be reasonably neglected. Based on the calculations shown in figure 8 and comparable studies found in literature [13, 34, an attenuation of $1 \mathrm{~dB} /$ canyon is proposed as an efficient but still reasonably accurate approach for $A_{\text {inter }}$ in more realistic situations. The geometrical data of Ghent (Belgium) and Södermalm (Sweden) 28] further show that one canyon per 100 meters is the most common canyon density. Because of the aforementioned reasons and given the complexity of the canyon structure in cities, the suggested broadband (and frequency independent) attenuation is $1 \mathrm{~dB}$ per $100 \mathrm{~m}$. Additionally, accounting for possible absorption at roof tops, e.g. the presence of green roofs that were shown to be an efficient noise reducing measure before [29], a maximum $5 \mathrm{~dB}$ is recommended in this model. This limit implicitly assumes that statistically speaking in downward refracting conditions no additional intermediate building will cut the curved propagation path once the distance reached $500 \mathrm{~m}$.

\section{Comparison with measurement}

An inner city noise measurement network in Ghent (Belgium) with microphone nodes 30 placed at both shielded and directly exposed locations is used as a first validation of the current engineering model. At these locations, road traffic noise was the main source of environmental noise exposure. The officially approved noise maps made for the agglomeration of Ghent in the framework of the END were used as the parent noise map. The same traffic intensity and traffic composition database (i.e. a combination of traffic counts and traffic flow modeling) as used for the END map was used for the background noise mapping calculations. The building coordinates and the heights are 


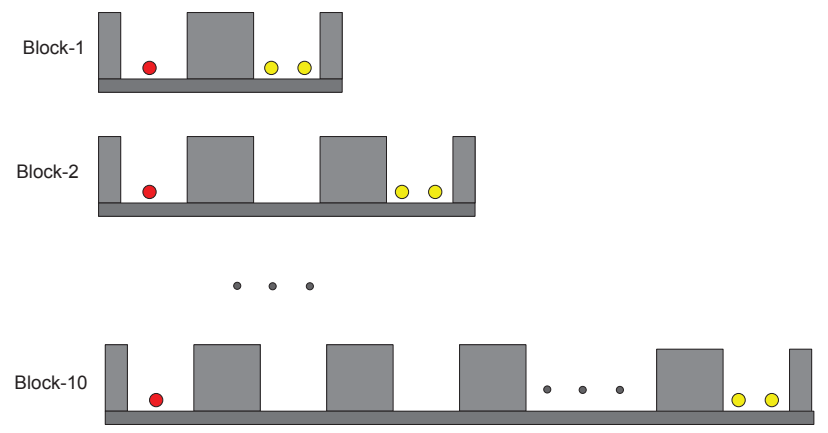

(a) Configuration

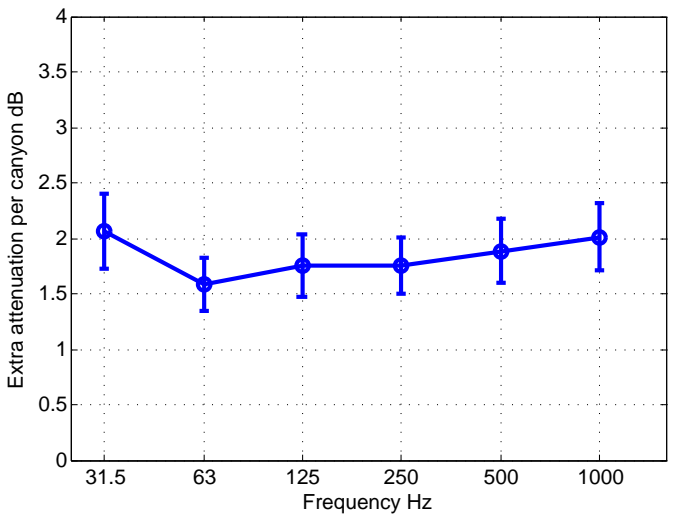

(b) Attenuation per canyon

Figure 8: Attenuation caused by multiple intermediate buildings of equal height, with $W_{s}=W_{r}=W_{i}=$ $10 \mathrm{~m}, H_{s}=H_{i}=H_{r}=9.6 \mathrm{~m}$. (a) Configuration; (b) Attenuation per canyon relative to no-canyon case. The error bar is the standard error extracted from all receivers in all configurations.

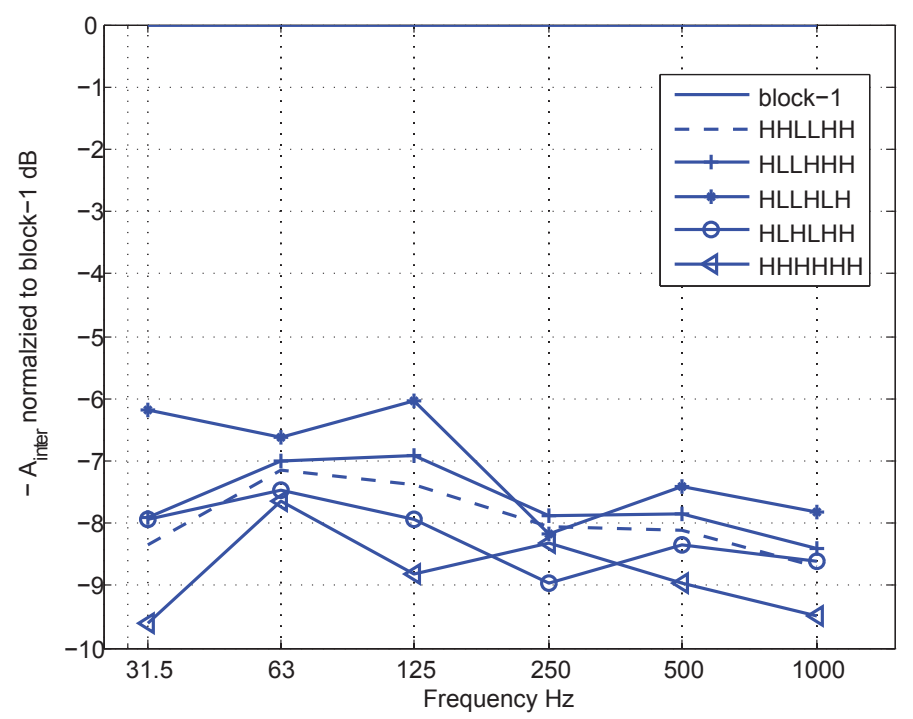

Figure 9: Intermediate canyon effect for different variations in the sequence of heights of intermediate buildings between source and receiver. See Fig. 8 for a description of Block-1. The curves are relative to the attenuation of a single large building of equal total length. 
extracted from a GIS system. The mean height of the buildings in the calculation zone is 10.9 $\mathrm{m}$ with $\mathrm{std}=4.50 \mathrm{~m}$. The quiet façade nodes are all located in residential zones inside the city and could be affected by human activities other than traffic noise. Therefore the time window was limited to 9:00 to 17:00 when the inhabitants of the house where the measurement equipment is installed are at work and traffic noise levels are considerable. For simplicity this value is still referred to as $L_{d a y}$. The measurement locations are chosen in such a way that continuous noise sources such as ventilation or cooling units are not present. Finally, a long observation period is considered to average out occasional gardening, cleaning, or rebuilding activities. Measured data during 90 days lead to convergence of the energetically averaged $L_{d a y}$ noise exposure indicator at all locations considered. All traffic noise sources up to $1500 \mathrm{~m}$ from each receiver were considered in the calculations. Figure 10 shows 9 measurement positions used for the validation and their locations on the map. The measurements, the END-reported noise levels and the predicted levels based on the background noise mapping concept are shown in figure 11. Since the distance from the measurement microphones to the wall is in most cases less than $20 \mathrm{~cm}, 3 \mathrm{~dB}$ is subtracted from the measurement to account for the coherence between the direct and reflected waves near the façades for very low frequencies $(<250 \mathrm{~Hz})$ 31, 32. Position 6, 8, and 9 are inside an enclosed yard and other positions are at a directly exposed façades. The sound spectrum at the totally shielded positions are shown in figure $12,13,14$. The results show that the noise levels of $L_{d a y}$ calculated by the END noise map are close to the measured levels in the directly exposed façades(except for position 4), but clearly underestimate levels at the shielded façades. In position 9, this difference exceeds $14 \mathrm{dBA}$. At shielded locations, the $A_{\text {can }}$ term significantly improves the noise level spectrum predictions at low frequencies but not at high frequencies. Adding a turbulent scattering contribution becomes therefore essential and the engineering model as described in detail in [10] was used. Applying the background noise mapping concept still leads to an underestimation at position 8, although an important improvement is made compared to the END map. At this specific small enclosed courtyard in the traffic free inner city, traffic noise hardly contributes to the observed noise level during the day.

At the most exposed façades, some difference between the measurements and the calculated levels for the END map could be attributed to e.g. inaccuracies in traffic data. Also the measurement error should be mentioned, which is expected to be below $2 \mathrm{dBA}$ for road traffic dominated environmental noise exposure [30].

\section{Conclusions}

An engineering model for improving noise level predictions at shielded locations in an urban noise map is presented, based on a large set of $2 \mathrm{D}$ full-wave numerical calculations of canyon-to-canyon propagation. The engineering model for background noise mapping proposed here can be used to improve existing noise maps with a poor prediction at shielded zones. In this model, different attenuation terms, $A_{b a r}, A_{c a n}$, and $A_{\text {inter }}$ are quantified separately, which opens possibilities to add more correction terms, such as terms to explicitly account for refraction by wind and temperature gradients. Another advantage is that the inputs of the model are only geometrical parameters of the canyons, buildings, sources and receivers. Such parameters are easily derived from common GIS systems. A comparison between predicted levels and long-term measurements, shows that the 


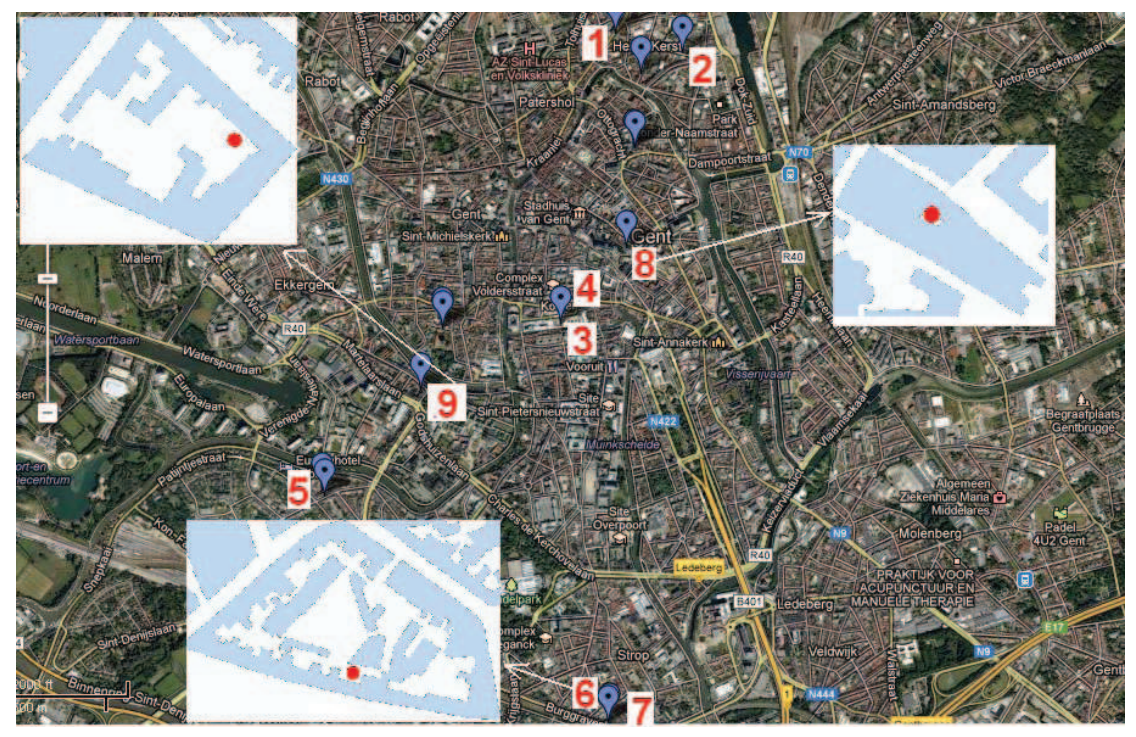

Figure 10: Measurement positions of Ghent city, Belgium.

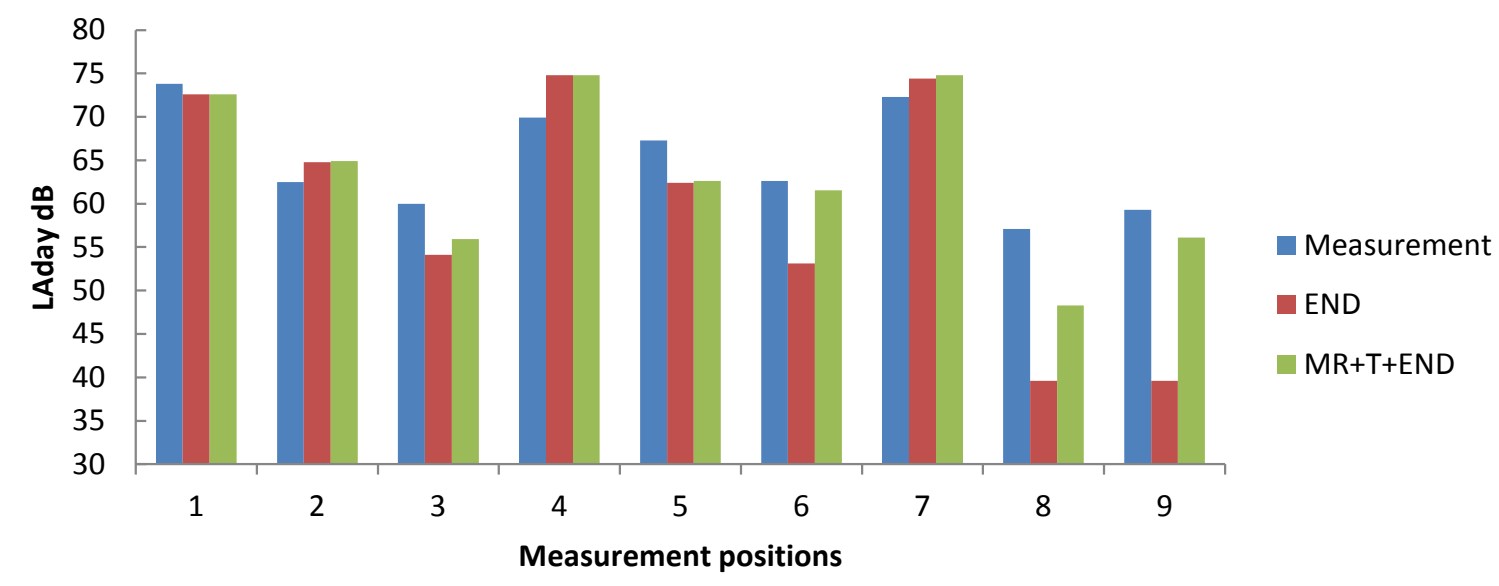

Figure 11: Comparison of predicted level and measurement. The legends "Measurement" indicates the measured $L_{d a y}$; "END" is the $L_{d a y}$ calculated for the END map; "MR+T+END" uses the background noise mapping concept, including multiple reflections and the turbulence scattering model [10] applied to the END map predictions. 


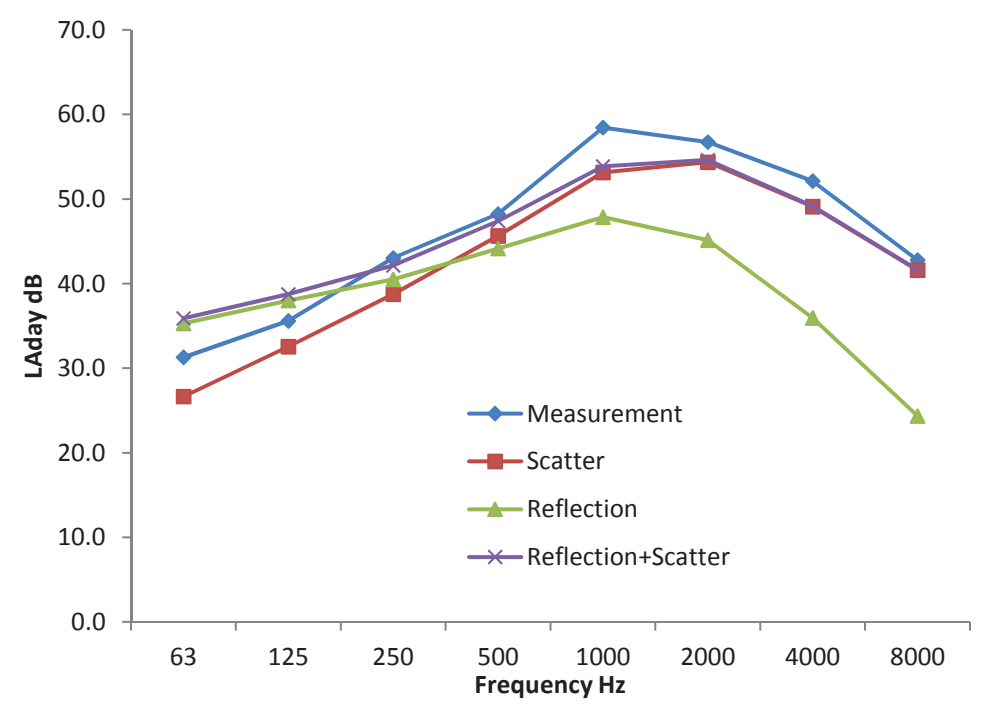

Figure 12: Spectrum of position 6. Legend "Scatter" is calculated by the model in [10]; "Reflection" is calculated by equation (2); "Reflection+Scatter" is calculated by equation (1) with $C_{t}^{2}=0.4 K^{2} / \mathrm{m}^{2 / 3}$ and $C_{v}^{2}=1.2 \mathrm{~m}^{4 / 3} / \mathrm{s}^{2} . C_{v}$ and $C_{t}$ are the structure parameters of velocity and temperature fluctuations, respectively, describing their partial turbulence strengths.

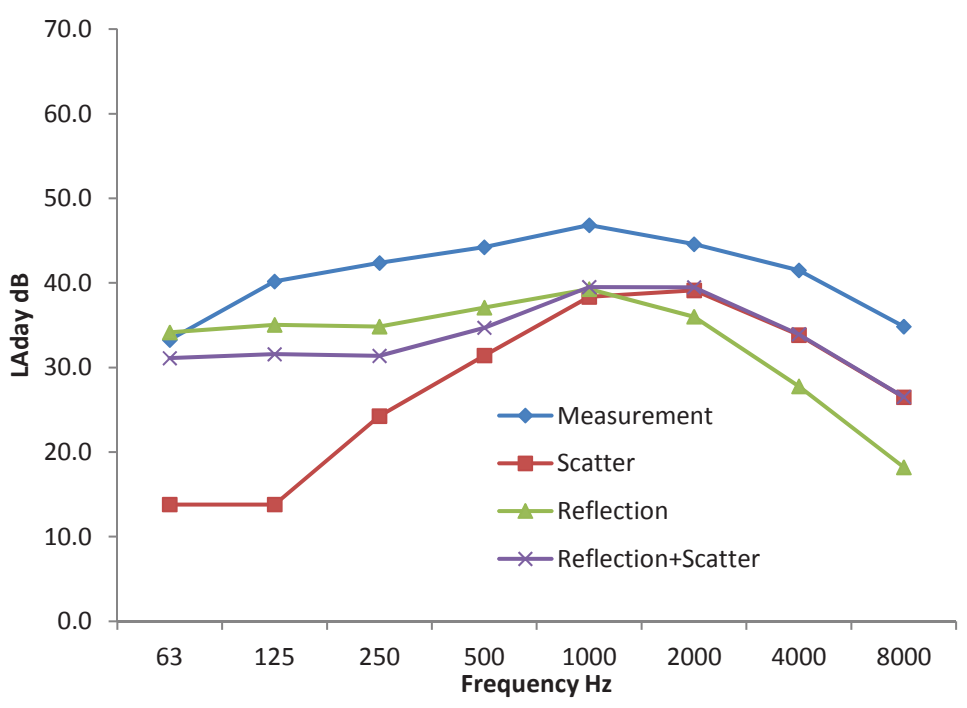

Figure 13: Spectrum of position 8. 


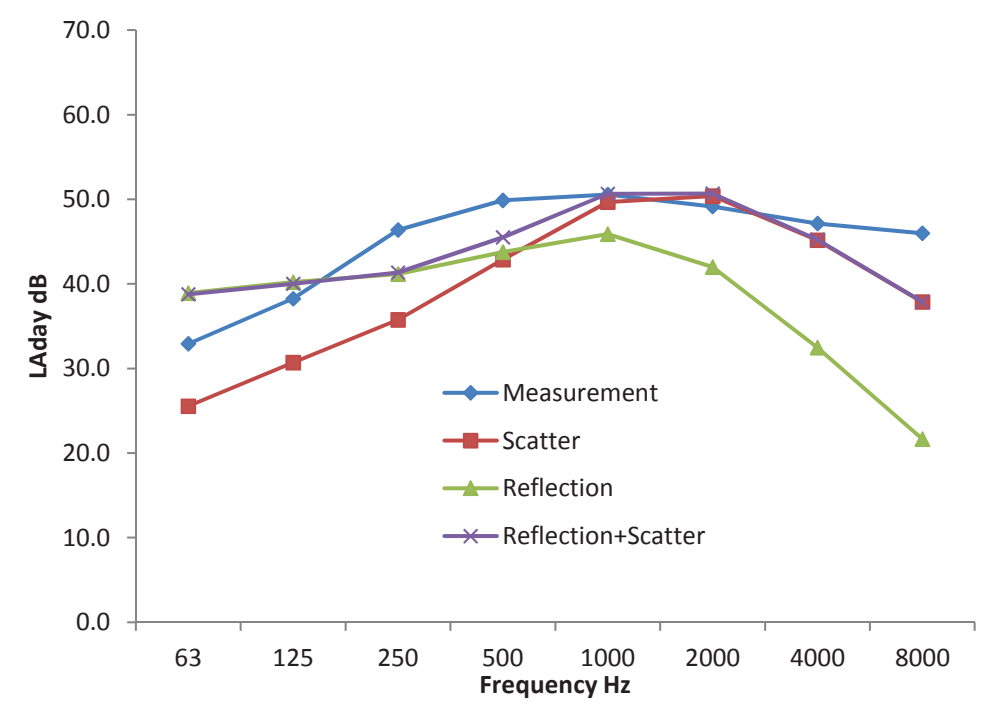

Figure 14: Spectrum of position 9.

model performs well in predicting total $L_{d a y}$ and the compatibility of the background noise mapping concept with existing noise maps is illustrated. Especially at shielded building façades, predictions are strongly improved.

\section{Acknowledgment}

We gratefully appreciate the financial support of the Life+ program of the European Community (project QSIDE, LIFE09 ENV/NL/000423).

\section{Appendix A: Analytic form for $A_{\text {can,flat }}$}

To derive the general form of the analytic expression that will be fitted to the numerical results, diffraction over the central building from multiple image sources in the source canyon to multiple image receivers in the receiver canyon is studied. The total sound pressure at the receiver caused by all of these propagation paths can be summed incoherently. The total contribution is:

$$
\sum_{i=0}^{\infty} \sum_{j=0}^{\infty}\left|p_{i, j}\right|^{2}=\left|p_{0,0}\right|^{2}+\sum_{i=1}^{\infty}\left|p_{i, 0}\right|^{2}+\sum_{j=1}^{\infty}\left|p_{0, j}\right|^{2}+\sum_{i=1}^{\infty} \sum_{j=1}^{\infty}\left|p_{i, j}\right|^{2}
$$

where the subscript indicates the position of the source, the receiver and the image sources and image receivers, i.e. $i=0$ indicates the source position and $j=0$ indicates the receiver position; the $i=1,2,3 \ldots$ indicates different image sources; similarly, $j=1,2,3 \ldots$ indicates different image receivers. $\left|p_{0,0}\right|^{2}$ contains the part of the squared sound pressure at the receiver, emitted by the source and diffracted directly over the building. This term is included in $A_{b a r, f l a t} . \quad \sum_{i=1}^{\infty}\left|p_{i, 0}\right|^{2}$ indicates the total squared sound pressure at the receiver position, emitted by all the image sources. $\sum_{j=1}^{\infty}\left|p_{0, j}\right|^{2}$ indicates the squared sound pressure emitted by the source and received by all the 
image receivers (i.e. located at the image receiver positions). $\sum_{i=1}^{\infty} \sum_{j=1}^{\infty}\left|p_{i, j}\right|^{2}$ indicates the total sound pressure square at all the image receiver positions, emitting by all the image sources. For each of these three terms, an analytic expression is derived below.

\section{A.1 Analytic solutions of $\sum_{i=1}^{\infty}\left|p_{i, 0}\right|^{2}$ and $\sum_{j=1}^{\infty}\left|p_{0, j}\right|^{2}$}

Image sources will occur in the direction away from the intermediate building and in the direction of this building. Because the diffraction angle is much larger and the distance is comparable for the latter set, it can easily be shown that these can be neglected. Thus the derivation can focus on the image sources positioned away from the intermediate building. A few assumptions are listed before hand. The first assumption is that the buildings are not very low. Accordingly, the decay caused by the finite size of the reflecting surface that could be expressed as a decaying overlap between the surface and the Fresnel zone can be ignored even after many reflections. This decay will be considered in a fitting coefficient later. A second assumption is that the building height of the source canyon and receiver canyon are the same. As a result, the image sources can reach the receiver or image receivers by double diffraction. Afterwards, other conditions such as, $H_{s}<H_{i}, H_{r}<H_{i}, H_{s}>H_{i}$ and $H_{r}>H_{i}$ will be studied. With the above assumptions and equation (4), the square of the sound pressure generated by the $i$ th image source is: $\left|p_{i, 0}\right|^{2}=\left(\frac{0.37}{X_{i, 0,1}+0.37}\right)^{2}\left(\frac{0.37}{X_{i, 0,2}+0.37}\right)^{2}\left|p_{a t, L_{i}}\right|^{2}$. For a point source, the sound pressure at distance $L_{i}$ is $p_{a t, L_{i}}=\frac{\rho_{s}^{i} A}{4 \pi L_{i}} e^{-j k L_{i}}$, where $A$ is the amplitude and $\rho_{s}$ is the reflection coefficient in the source canyon. Note that in accordance with the diffraction theory $L_{i}$ is the shortest path between source and receiver around the diffracting elements. Then the sum of $p_{i, 0}$ is:

$$
\sum_{i=1}^{\infty}\left|p_{i, 0}\right|^{2}=\sum_{i=1}^{\infty}\left|\frac{\rho_{s}^{i} A}{4 \pi}\right|^{2}\left|\frac{1}{L_{i, 0}}\right|^{2}\left(\frac{0.37}{X_{1, i, 0}+0.37}\right)^{2}\left(\frac{0.37}{X_{2, i, 0}+0.37}\right)^{2}
$$

In a general case, $Y_{s}=M_{\nu s} \gamma_{s}=\sqrt{\frac{2 r_{s, i}\left(r_{r}+W_{i}\right)}{r_{s, i}+r_{r}+W_{i}}} \sqrt{3}\left(\cos \frac{2}{3} \phi_{s, i}-0.5\right)$. For $\phi_{s, i}$ in the range between 0 and $\pi / 2$, which is the shielded area, $\cos \frac{2}{3} \phi_{s, i}-0.5$ can be approximated by $0.5 \cos \phi_{s, i}$ which is calculated as $0.5 h_{1} / r_{s, i}$. It can easily be verified that this approximation introduces a very small error, $s t d=0.0056$ in more than 1500 checking samples. As a result, $Y_{s}$ and $Y_{r}$ can be simplified as:

$$
\begin{aligned}
Y_{s, i} & \approx \sqrt{\frac{2 r_{s, i}\left(r_{r}+W_{i}\right)}{r_{s, i}+r_{r}+W_{i}}} \frac{\sqrt{3}}{2} \cos \phi_{s, i}=\sqrt{\frac{2 r_{s, i}\left(r_{r}+W_{i}\right)}{r_{s, i}+r_{r}+W_{i}}} \frac{\sqrt{3}}{2} \frac{h_{1}}{r_{s, i}} ; \\
Y_{r, i} & \approx \sqrt{\frac{2 r_{r}\left(r_{s, i}+W_{i}\right)}{r_{s, i}+r_{r}+W_{i}}} \frac{\sqrt{3}}{2} \cos \phi_{r, i}=\sqrt{\frac{2 r_{r}\left(r_{s, i}+W_{i}\right)}{r_{s, i}+r_{r}+W_{i}}} \frac{\sqrt{3}}{2} \frac{h_{2}}{r_{r}} ;
\end{aligned}
$$

According to the diffraction theory, the factor B has to be multiplied with the smallest of the two $\mathrm{Y}$ terms. Therefore we have a closer look at the ratio $Y_{s, i} / Y_{r, i}$ which is $\frac{h_{1}}{h_{2}} \sqrt{\frac{r_{r}\left(r_{r}+W_{i}\right)}{r_{s, i}\left(r_{s, i}+W_{i}\right)}}$. Considering that the receiver is generally higher than the source and that all façades of buildings have the same height, $h_{1}>h_{2}$. In most cases it can be shown that this ratio is less than 1 after a few reflections, since $r_{s, i} \gg r_{r}$ is expected. For deducing an analytic form for $A_{c a n}^{f}$, all $Y_{s, i}$ are supposed to be less than $Y_{r}$. As a result, $X_{1, i, 0}=Y_{s, i} B$ and $X_{2, i, 0}=Y_{r}$. 
Let us now consider the second term in equation 16 which we call $C_{1 s}$ for convenience:

$$
\begin{aligned}
C_{1 s}=\left(\frac{0.37}{X_{2, i, 0}+0.37}\right)^{2} & \approx\left[\frac{0.37}{\sqrt{\frac{2 r_{r}\left(W_{i}+r_{s, i}\right)}{\lambda\left(r_{s, i}+W_{i}+r_{r}\right)} \frac{\sqrt{3}}{2}} \cos \phi_{r}+0.37}\right]^{2} \\
& \approx\left[\frac{0.37}{\sqrt{\frac{2 r_{r}}{\lambda}} \frac{\sqrt{3}}{2} \cos \phi_{r}+0.37}\right]^{2}
\end{aligned}
$$

which becomes independent of $\mathrm{i}$ when it can be assumed that $W_{i}+r_{s, i} \gg r_{r}$, which is the case for higher order reflections at least. The remaining part of equation 16 can be rewritten as:

$$
\begin{aligned}
\left|\frac{1}{L_{i, 0}}\right|^{2}\left(\frac{0.37}{X_{1, i, 0}+0.37}\right)^{2} & \approx\left[\frac{0.37}{\sqrt{\frac{2 r_{s, i} W_{i}}{\lambda\left(W_{i}+r_{s, i}\right)}} \frac{\sqrt{3}}{2} \cos \left(\phi_{s, i}\right) L_{i, 0}+0.37 L_{i, 0}}\right]^{2} \\
& =\left[\frac{1}{\sqrt{\frac{2 W_{i}\left(r_{s, i}+W_{i}+r_{r}\right)}{\lambda\left(r_{s, i}+W_{i}\right)}} \frac{\sqrt{3}}{0.74} h_{1} \sqrt{\frac{\left(r_{s, i}+W_{i}+r_{r}\right)}{r_{s, i}}}+\left(r_{s, i}+W_{i}+r_{r}\right)}\right]^{2}
\end{aligned}
$$

Again assuming that $r_{s, i}+W_{i} \gg r_{r}$, the first square root term simplifies and becomes independent of the reflection order $i . \sqrt{\frac{\left(r_{s, i}+W_{i}+r_{r}\right)}{r_{s, i}}}$ is difficult to handle but fortunately assuming that it is close to 1 introduces at most $3 \mathrm{~dB}$ of error for the $r_{s, i}$ and $W_{i}$ that can be expected in an urban setting. The reader should keep in mind that the purpose of this derivation is to extract an analytic form with coefficients that will be fitted on numerical simulation results. As a result of these approximations, the total sum of equation (16) is reduced to:

$$
\sum_{i=1}^{\infty}\left|p_{i, 0}\right|^{2}=C_{1 s} \sum_{i=1}^{\infty}\left|\frac{\rho_{s}^{i} A}{4 \pi}\right|^{2}\left(\frac{1}{\sqrt{\frac{2 W_{i}}{\lambda} \frac{\sqrt{3}}{0.74} h_{1}+r_{s, i}+W_{i}+r_{r}}}\right)^{2}
$$

The first term in the denominator is independent of the image source index $i$. This implies that the approximations made above boil down to assuming that the effect of increasing distance from the image source to the diffraction edge is neatly compensated by the effect of changing diffraction angle. To simplify the sum further it is now assumed that the source is positioned in the middle of the canyon and that when $r_{s, i}$ becomes large compared to the height of the canyon above the source $h_{1}$, its value can approximated by $r_{s, i} \approx D_{i}$, where $D_{i}=i * W_{s}+0.5 W_{s}$ is the horizontal distance from the $i$ th image source to the edge of the building façade. In this case, the sum is a special function:

$$
\sum_{i=1}^{\infty}\left|p_{i, 0}\right|^{2}=C_{1 s}\left|\frac{A}{4 \pi}\right|^{2} \frac{\rho_{s}^{2}}{W_{s}^{2}} \Phi\left(\rho_{s}^{2}, 2, \frac{C_{3 s}+W_{s}}{W_{s}}\right)
$$

where $C_{3 s}=\sqrt{\frac{2 W_{i}}{\lambda}} \frac{\sqrt{3}}{0.74} h_{1}+0.5 W_{s}+r_{r}+W_{i} . \Phi$ is the Hurwitz-Lerchi transcendent.

Until now it was assumed that the flanking building creating the source street canyon was very high so that all reflections were possible. However, when $H_{s}<H_{i}$, the sound emitting from some of the left image sources cannot contribute to the receiver and the contribution of the image sources 


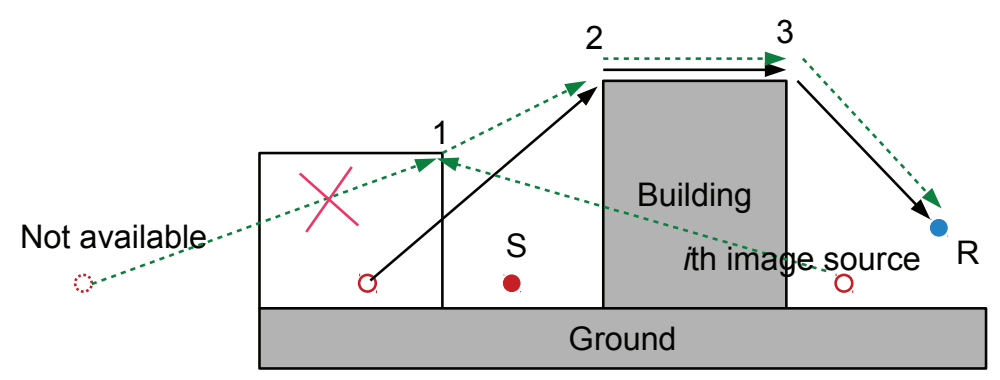

Figure 15: Demonstration of contributed image sources.

from the right side start becoming stronger. The sound will need to diffract three times to reach the receiver and follows the route "image source $\rightarrow 1 \rightarrow 2 \rightarrow 3 \rightarrow$ receiver" to reach the receiver position, as shown in figure 15 .

After one more diffraction, the sound power decreases significantly, which can be accordingly ignored. When $H_{s}$ is not much smaller than $H_{i}$, the sound from the first few important images sources can still reach the receiver position by double diffraction following the routine "image source $\rightarrow 2 \rightarrow 3 \rightarrow$ receiver", as shown in figure 15 . Under this condition and neglecting high order diffraction, the total contribution of the image sources is approximated by:

$$
\begin{aligned}
& \sum_{i=1}^{N}\left|p_{i, 0}\right|^{2}=\sum_{i=1}^{\infty}\left|p_{i, 0}\right|^{2}-\sum_{i=N+1}^{\infty}\left|p_{i, 0}\right|^{2} \\
= & C_{1 s}\left|\frac{A}{4 \pi}\right|^{2} \frac{\rho_{s}^{2}}{W_{s}^{2}} \Phi\left(\rho_{s}^{2}, 2, \frac{C_{3 s}+W_{s}}{W_{s}}\right)-C_{1 s}\left|\frac{A}{4 \pi}\right|^{2} \frac{\rho_{s}^{2(N+1)}}{W_{s}^{2}} \Phi\left(\rho_{s}^{2}, 2, N+1+\frac{C_{3 s}}{W_{s}}\right)
\end{aligned}
$$

where $\mathrm{N}$ is the number of images sources which can reach the receiver by only two diffraction. As expected, the higher $H_{s}$ is the larger $\mathrm{N}$ is. When $H_{s}=H_{i}, \mathrm{~N}$ becomes infinite. The number of visible image sources, $\mathrm{N}$, is the most important parameter to determine the difference between the level calculated using equation (23) and equation (22). Other parameters, such as $W_{s}, W_{i}$ and $\lambda$ can still affect this level difference slightly. To avoid calculating $\mathrm{N}$ for every source position in the canyon, $\mathrm{N}$ is proposed to use the assumption that the source is in the middle of the canyon and categorize situations according to the ratio of $\left(H_{s}-h_{s}\right) /\left(H_{i}-h_{s}\right)$. The relation can be written as: $H_{s}-h_{s}=$ $\frac{2 N-1}{2 N+1}\left(H_{i}-h_{s}\right)$ for a source in the middle of the source canyon. Specifically, when $N=[1,2,3, \cdots]$ corresponds to the ratio $\left(H_{s}-h_{s}\right) /\left(H_{i}-h_{s}\right) \leq[1 / 3,3 / 5,5 / 7 \cdots]$. When $\left(H_{s}-h_{s}\right) /\left(H_{i}-h_{s}\right) \leq 1 / 3$, no image sources are available from the left side and the canyon effect can be neglected. $L_{h s}$ is set to $-\infty$. When $1 / 3<\left(H_{s}-h_{s}\right) /\left(H_{i}-h_{s}\right) \leq 3 / 5$, only the first image source from the left side can contribute and $\sum\left|p_{i, 0}\right|^{2}=10^{\left(-0.1 A_{\text {bar,flat }}\right)}$ with the "source position" being at the first image source; when $3 / 5<\left(H_{s}-h_{s}\right) /\left(H_{i}-h_{s}\right)$, more than one image source from the left side are available, an approximation of the level difference between equation 23 and equation 22 is: $L_{h s}=-6.17\left(1-\frac{H_{s}-h_{s}}{H_{i}-h_{s}}\right)\left[1-1.37 \log _{10}\left(\frac{\sqrt{\lambda W_{s}}}{W_{i}}\right)\right] \mathrm{dB}$ which is fitted on the condition of $W_{s} \in$ $[15,100], W_{i} \in[20,500], H_{i}=18 \mathrm{~m}$ and Frequency $\in[60,8000] \mathrm{Hz}$. When $\frac{H_{s}-h_{s}}{H_{i}-h_{s}}>1, L_{h s}=0$.

When $H_{s}>H_{i}$, in most cases the important contribution comes from the sound from the image 
sources diffracting twice over the middle building. In some extreme cases, when $H_{s}$ is high, $H_{i}$ is low and $W_{i}$ is narrow, sound could reach the receiver after only one reflection on the edge of the building of height $H_{s}$. Since this condition is not common, its effect is ignored when extracting the analytic form of the functions used for fitting. Without the numerical constant, the fitting formula for $\sum_{i=1}^{\infty}\left|p_{i, 0}\right|^{2}$ is:

$$
\sum_{i=1}^{\infty}\left|p_{i, 0}\right|^{2} \approx F(1)\left[C_{1 s} \frac{\rho_{s}^{2}}{W_{s}^{2}} \Phi\left(\rho_{s}^{2}, 2, \frac{C_{3 s}+W_{s}}{W_{s}}\right) 10^{0.1 L_{h s}}\right] \quad \text { if } \quad \frac{3}{5}<\frac{H_{s}-h_{s}}{H_{i}-h_{s}} \leq 1
$$

where $F(1)$ is a fitting coefficient. Similarly, the $\sum_{j=1}^{\infty}\left|p_{0, j}\right|^{2}$ could also be obtained.

$$
\sum_{j=1}^{\infty}\left|p_{0, j}\right|^{2} \approx F(2)\left[C_{1 r} \frac{\rho_{r}^{2}}{W_{r}^{2}} \Phi\left(\rho_{r}^{2}, 2, \frac{C_{3 r}+W_{r}}{W_{r}}\right) 10^{0.1 L_{h r}}\right] \quad \text { if } \quad \frac{3}{5}<\frac{H_{r}-h_{r}}{H_{i}-h_{r}} \leq 1
$$

where $F(2)$ is the fitting coefficient, $\rho_{r}$ is the average reflection coefficient of the façade in the receiver canyon and other parameters are as follows:

$$
\begin{aligned}
L_{h r} & =-6.17\left(1-\frac{H_{r}-h_{r}}{H_{i}-h_{r}}\right)\left[1-1.37 \log _{10}\left(\frac{\sqrt{\lambda W_{r}}}{W_{i}}\right)\right] \\
C_{1 r} & \approx\left(\frac{0.37}{\sqrt{\frac{2 r_{s}}{\lambda}} \frac{\sqrt{3}}{2} \cos \phi_{s}+0.37}\right)^{2} \\
C_{3 r} & =\sqrt{\frac{2 W_{i}}{\lambda}} \frac{\sqrt{3}}{0.74} h_{2}+0.5 W_{r}+r_{s}+W_{i}
\end{aligned}
$$

Similarly, when $\frac{H_{r}-h_{r}}{H_{i}-h_{r}}<1 / 3$, the canyon effect is neglected and $L_{h r}$ is set to $-\infty ;$ when $1 / 3<$

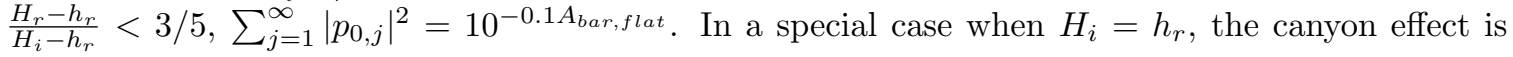
neglected as well.

To quantify the effect of the finite size of an object on the amount of the reflected acoustic energy, the envelope of the object and the Fresnel ellipsoid should be calculated. The source height of a vehicle is often close to the ground which implies that half of the section of the ellipsoids is below the building façade. If the reflections on the ground are considered, its contribution can be treated as an image source. As a result, the Fresnel zone can only cause decay when the radius of the Fresnel ellipsoids is greater than $H_{s}$ or $H_{i}$. If the radius $\sqrt{\lambda D} / 2 \leq H_{s}$, the sound energy will be totally reflected, where $\mathrm{D}$ equals twice the distance of the image source which is $D=2\left(i W_{s}+0.5 W_{s}\right)$. If $H_{s}=12 \mathrm{~m}$ (corresponding to a 3-floors building), $W_{s}=15 \mathrm{~m}$ (appears frequently in Gent), the Fresnel zone starts to cause decay after 10 and 112 reflection for $\lambda=3.4 \mathrm{~m}$ (corresponding to $100 \mathrm{~Hz}$ ) and $\lambda=0.34 \mathrm{~m}$ (corresponding to $1000 \mathrm{~Hz}$ ) respectively. It can be concluded that the decay speed is much less than the decay caused by the absorption of the façade. which decays by power function and the absorption starts to decay from the first reflection.

For the receiver canyon, it is difficult to make accurate estimation considering the receiver height is often at $4 \mathrm{~m}$. Suppose $H_{s}=10 \mathrm{~m}, H_{i}=10 \mathrm{~m}, W_{s}=12 \mathrm{~m}$, The decay starts from 2 for $100 \mathrm{~Hz}$ and 20 for $1000 \mathrm{~Hz}$. Although the decay due to the Fresnel ellipsoid depends differently on the reflection area, it is decided to include its effect by increasing the average contribute of the façade. 


\section{A.2 Approximation of $\sum_{i=1}^{\infty} \sum_{j=1}^{\infty}\left|p_{i, j}\right|^{2}$}

The double sum can be written as a sum of single sums for the source canyons for example. It is already known that the sum over all image sources results in the Hurwitz-Lerchi transcendent, but it is not possible to convert the sum over these special functions to a closed form. In the next step, the Hurwitz-Lerchi transcendent $\Phi$ is approximated by $G\left(\rho_{s}, x\right)=K \rho_{s}^{2} / x^{2}$. In the region $x \in(5,20]$, $\rho_{s} \in[0.8,1]$, this approximation with $K=1.59$ results in a mean squared error of 0.0034 , which is acceptable. Because the solution of every sum $\sum_{i=j}^{\infty}\left|p_{i, j}\right|^{2}$ is similar as equation 22 , the double sum can be generally written as:

$$
\sum_{i=j}^{\infty} \sum_{i=1}^{\infty}\left|p_{i, j}\right|^{2}=10^{L_{h s}}\left|\frac{A}{4 \pi}\right|^{2} \sum_{j=1}^{\infty} C_{1 s, j} \frac{\rho_{s}^{2} \rho_{r}^{2 j}}{W_{s}^{2}} \Phi\left(\rho_{s}^{2}, 2, \frac{C_{3 s, j}+W_{s}}{W_{s}}\right)
$$

where $C_{3 s, j}=\sqrt{\frac{2 W_{i}}{\lambda}} \frac{\sqrt{3}}{0.74} h_{1}+0.5 W_{s}+r_{r, j}+W_{i}$. It should be mentioned that while deriving this equation, it was assumed that $r_{s, i} \gg r_{r, j}$ which may not hold for high order receiver reflections. According to the approximation mentioned in this section, equation 26 changes to:

$$
\sum_{i=j}^{\infty} \sum_{i=1}^{\infty}\left|p_{i, j}\right|^{2}=1.59 \rho_{s}^{2} L_{h s}\left|\frac{A}{4 \pi}\right|^{2} \sum_{j=1}^{\infty}\left(\frac{0.37}{\sqrt{\frac{2 r_{r, j}}{\lambda} \frac{\sqrt{3}}{2}} \cos \phi_{r, j}+0.37}\right)^{2}\left(\frac{1}{C_{3 s, j}+W_{s}}\right)^{2}
$$

For high order image receivers, $\left(\frac{0.37}{\sqrt{\frac{2 r_{r, j}}{\lambda} \frac{\sqrt{3}}{2}} \cos \phi_{r, j}+0.37}\right)^{2}=\left(\frac{0.37}{3.31 h_{1} / \sqrt{\lambda r_{r, j}}+0.37}\right)^{2} \rightarrow 1$. As a result, the above equation approximates to:

$$
\begin{aligned}
\sum_{i=j}^{\infty} \sum_{i=1}^{\infty}\left|p_{i, j}\right|^{2} & <1.59 \rho_{s}^{2}\left|\frac{A}{4 \pi}\right|^{2} \sum_{j=1}^{\infty}\left(\frac{1}{C_{3 s, j}+W_{s}}\right)^{2} \\
& =\left|\frac{A}{4 \pi}\right|^{2} 1.59 \rho_{s}^{2} \frac{\rho_{r}^{2}}{W_{r}^{2}} \Phi\left(\rho_{r}^{2}, 2, \frac{3.31 h_{1} / \sqrt{\lambda}+1.5 W_{s}+W_{i}+1.5 W_{r}}{W_{r}}\right) \\
& \approx\left|\frac{A}{4 \pi}\right|^{2}\left(\frac{1.59 \rho_{s} \rho_{r}}{3.31 h_{1} / \sqrt{\lambda}+1.5 W_{s}+W_{i}+1.5 W_{r}}\right)^{2}
\end{aligned}
$$

The asymmetry is caused by the above assumption. If we calculate the double sum from the receiver canyon, a similar form could be achieved only replacing " $h_{1}$ " by " $h_{2}$ ". To moderate this error, the average of the two calculations is used to approximate the double sum by:

$$
\sum_{i=j}^{\infty} \sum_{i=1}^{\infty}\left|p_{i, j}\right|^{2} \approx\left|\frac{A}{4 \pi}\right|^{2} \frac{\left(1.59 \rho_{s} \rho_{r}\right)^{2}}{\left(3.31 h_{1} / \sqrt{\lambda}+1.5 W_{s}+W_{i}+1.5 W_{r}\right)\left(3.31 h_{2} / \sqrt{\lambda}+1.5 W_{s}+W_{i}+1.5 W_{r}\right)}
$$

\section{References}

[1] E. Öhrström, A. Skånberg, H. Svensson, and A. Gidlöf-Gunnarsson. Effects of road traffic noise and the benefit of access to quietness. J. Sound Vib., 295:40-59, 2005. 
[2] E. Öhrström. Psycho-social effects of traffic noise exposure. J. Sound Vib., 151(3):513-517, 1991.

[3] Y. de Kluizenaar, E. Salomons, S. Janssen, F. van Lenthe, H. Vos, H. Zhou, H. M. E. Miedema, and J. Mackenbach. Urban road traffic noise and annoyance: The effect of a quiet façades. $J$. Acoust. Soc. Am., 130(4):1936-1942, 2011.

[4] T. Van Renterghem and D. Botteldooren. Focused study on the quiet side effect in dwellings highly exposed to road traffic noise. International Journal of Environmental Research and Public Health, 9(12):4292-4310, November 2012.

[5] F. Van den Berg, C. Schoonebeek, and M. Hillebregt. On the definitions of quiet façades and quiet urban areas. In Proc. of Euronoise 2012, Prague, Prague, Czech, August 2012.

[6] M. Ögren and J. Forssén. Modelling of a city canyon problem in a turbulent atmosphere using an equivalent sources approach. Appl. Acoust., 65:629-642, 2004.

[7] M. Hornikx and J. Forssén. The 2.5-dimensional equivalent sources method for directly exposed and shielded urban canyons. J. Acoust. Soc. Am., 122:2532-2541, 2007.

[8] T. Van Renterghem and D. Botteldooren. Prediction-step staggered-in-time fdtd: An efficient numerical scheme to solve the linearised equations of fluid dynamics in outdoor sound propagation. Appl. Acoust., 68:201-216, 2007.

[9] D. Botteldooren. Finite-difference time-domain simulation of low-frequency room acoustic problems. J. Acoust. Soc. Am., 98:3302-3308, 1995.

[10] J. Forssén, Maarten Hornikx, T. Van Renterghem, W. Wei, D. Botteldooren, and M. Ögren. Urban background noise mapping: the turbulence scattering model. Submitted to Acta Acust. Acust., 2013.

[11] J. Defrance, E. Salomons, I. Noordhoek, D. Heimann, B. Plovsing, G. Watts, H. Jonasson, X. Zhang, E. Premat, I. Schmich, F. Aballea, M. Baulac, and F. de Roo. Outdoor sound propagation reference model developed in the european harmonoise project. Acta Acust. Acust., 93:213-227, 2007.

[12] Environmental noise and the cnossos-eu initiative, 2012.

[13] T. Van Renterghem, E. Salomons, and D. Botteldooren. Parameter study of sound propagation between city canyons with coupled fdtd-pe model. Appl. Acoust., 67(9):487-510, 2006.

[14] M. Hornikx, J. Forssén, T. Van Renterghem, D. Botteldooren, W. Wei, M. Ögren, and Erik Salomons. Urban background noise mapping: the multiple-reflection correction term. Acta Acust. Acust. 100(2):293-305, 2014

[15] J. Kang. Urban Sound Environment. Taylor \& Francis incorporating, Spon, London, 2007.

[16] C. Torrence and G. Compo. A practical guide to wavelet analysis. B. Am. Meteorol. Soc., 79:61-78, 1998.

[17] M. Hornikx and J. Forssén. A scalemodel study of parallel urban canyons. Acta Acust. Acust., 94:265-281, 2008.

[18] T. Van Renterghem, E. Salomons, and D. Botteldooren. Efficient fdtd-pe model for sound propagation in situations with complex obstacles and wind profiles. Acta Acust. Acust., 91:671$679,2005$. 
[19] K. Heutschi. Calculation of reflections in an urban environment. Acta Acust. Acust., 95:644-652, 2009.

[20] T. Van Renterghem and D. Botteldooren. Meteorological influence on sound propagation between adjacent city canyons: A real-life experiment. J. Acoust. Soc. Am., 127(6):3335-3346, 2010 .

[21] A. Pierce. Diffraction of sound around corners and over wide barriers. J. Acoust. Soc. Am., 55(5):941-955, 1974.

[22] A. Pierce. Acoustics: An Introduction to Its Pysical Principles and Applications. Acoustical Society of America, New York, USA, 1989.

[23] T. Kawai. Sound diffraction by a many-sided barrier or pillar. J. Sound Vib., 79(2):229-242, 1981.

[24] H. Kim, J. Kim, H. Kang, B. Kim, and S. Kim. Sound diffraction by multiple wedges and thin screens. Appl. Acoust., 66(9):1102-1119, September 2005.

[25] N.N. Lebedev. Special Functions and their Applications. Dover Publications, Inc., 1972.

[26] T. Van Renterghem and D. Botteldooren. The importance of roof shape for road traffic noise shielding in the urban environment. J. Sound Vib., 329(9):1422-1434, 2010.

[27] M. Ögren and J. Forssén. Road traffic noise propagation between two dimensional city canyons using an equivalent source approach. Acta Acust. Acust., 90:293-300, 2004.

[28] P. Thorsson, M. Ögren, and W. Kropp. Noise levels on the shielded side in cities using a flat city model. Appl. Acoust., 65:313-323, 2004.

[29] T. Van Renterghem and D. Botteldooren. In-situ measurements of sound propagating over extensive green roofs. Building and Environment, 46(3):729-738, 2011.

[30] T. Van Renterghem, P. Thomas, F. Dominguez, S. Dauwe, A. Touhafi, B. Dhoedt, and D. Botteldooren. On the ability of consumer electronics microphones for environmental noise monitoring. Journal of environmental monitoring: JEM, 13(3):544-552, March 2011. PMID: 21157618.

[31] G. Memoli and M. Paviotti and S. Kephalopoulos and G. Licitra. Testing the acoustical corrections for reflections on a façade. Appl. Acoust., 69(6):479-495, 2008.

[32] S. Tang, K. Li. The prediction of façade effects from a point source above an impedance ground. J. Acoust. Soc. Am., 110(1):278-288, 2001.

[33] M. Almgren. Simulation by using a curved ground scale model of outdoor sound propagation under the influence of a constant sound speed gradient. J. Sound Vib., 118(2):353-370, 1987.

[34] M. Hornikx and K. Conen and T. Hooff and B. Blocken. The influence of the urban flow field modelling approach on computing sound propagation over the urban roof level. AIA-DAGA 2013 Merano, Italy, (1):252-255, 2013.

[35] M. Schiff and M. Hornikx and J. Forssén. Excess attenuation for sound propagation over an urban canyon. Appl. Acoust., 71(6):510-517, 2010. 